\title{
Hematúria Microscópica Assintomática: Qual a Melhor Forma de Abordagem?
}

\section{Asymptomatic Microscopic Hematuria. What is the Best Approach?}

\section{Diana da Rocha, ${ }^{1}$ Fábia Martins ${ }^{2}$}

\section{Resumo}

Introdução: A hematúria microscópica assintomática (HMA) é comumente encontrada de forma incidental nos Cuidados de Saúde Primários (CSP). A importância deste achado é secundária ao potencial risco subjacente de patologia clinicamente significativa, incluindo malignidade, urolitíase e doença renal médica. Enquanto a hematúria macroscópica é reconhecida como um sinal que deve levar ao encaminhamento urológico para avaliação, a investigação recomendada da hematúria microscópica e, em particular, assintomática é inconsistente entre as diretrizes disponíveis.

O objetivo foi rever as principais diretrizes sobre a avaliação e abordagem da HMA e disponibilização de algoritmo de abordagem

Métodos: Pesquisa bibliográfica de artigos científicos nas bases de dados PubMed, UpToDate e Cochrane Library, publicados nos últimos 5 anos, utilizando a combinação dos termos MeSH "Hematuria/diagnosis", em inglês e português. Consulta das guidelines da American Urological Association (AUA) e da Associação Portuguesa de Urologia.

Resultados: Obtidos 733 artigos da pesquisa. Foram selecionados cinco artigos de revisão, pela adequação dos mesmos ao objetivo principal. A revisão sistemática de 2020, que contempla as guidelines mais recentes da AUA, mostra que a história cuidadosa e exame físico são recomendados por todas as diretrizes revisadas, permitindo identificar potenciais etiologias benignas que, se confirmadas, podem evitar a necessidade de avaliação adicional. Laboratorialmente, é recomendada, além da análise sumária de urina, a determinação da função renal na exclusão de patologia nefrológica. Há consenso pelas diretrizes de que a cistoscopia deve ser realizada.

Relativamente à avaliação radiológica, a diretriz mais atual baseia-se na estratificação do risco de malignidade no pedido de exames complementares.

Discussão: As diretrizes atuais variam em relação a detalhes importantes como a definição de HMA, o método ideal para avaliação radiológica e o papel da citologia urinária. É importante salientar que estas variações refletem a ausência de evidências de nível I sobre o assunto.

Palavras-chave: Algoritmos; Doenças Urológicas; Hematúria

1 - USF Progresso e Saúde, ACES Baixo Mondego, Coimbra, Portugal

2 - UCSP Cantanhede, ACES Baixo Mondego, Coimbra, Portugal
Abstract

Introduction: Asymptomatic microscopic hematuria $(A M H)$ is commonly found in Primary Health Care (PHC). The importance of this finding is secondary to the potential underlying risk of clinically significant pathology, including malignancy, urolithiasis and medicalkidney disease.

While macroscopic hematuria is recognized as a sign that should lead to urological referral for evaluation, the recommended investigation of microscopic and, in particular, asymptomatic hematuria is inconsistent among the available guidelines.

Our objective was to review the main guidelines on the assessment and approach of $\mathrm{AMH}$ and to provide an approach algorithm

Methods: Bibliographic search of scientific articles in the PubMed, UpToDate and Cochrane Library databases, published in the last 5 years, using the combination of MeSH terms "Hematuria / diagnosis", in English and Portuguese. Consultation of the guidelines of the American Urology Association (AUA) and the Portuguese Urology Association.

Results: A total of 733 research articles were obtained. Five review articles were selected, due to their adequacy to the main objective. The 2020 systematic review, which includes the most recent AUA guidelines, shows that careful history and physical examination are recommended by all revised guidelines, allowing to identify potential benign etiologies that, if confirmed, may avoid the need to proceed with an assessment additional.

In addition to urinary sediment, the determination of renal function is recommended in the exclusion of renal pathology. There is consensus by the guidelines that cystoscopy should be performed. Regarding radiological evaluation, the most current guideline is based on stratifying the risk of malignancy when requesting additional tests.

Discussion: Current guidelines differ on important details such as the definition of $\mathrm{AMH}$, the ideal method for radiological evaluation and the role of urine cytology. It is important to note that these variations reflect the absence of level I evidence on the subject.

\section{Keywords: Algorithms; Hematuria; Urologic Diseases}

\section{Introdução}

A hematúria está entre os diagnósticos mais comumente encontrados em urologia (>20\% das avaliações urológicas). ${ }^{1-3}$ Apesar da ausência de recomendações para a sua pesquisa, milhões de utentes realizam o teste de urina com fita reagente e exames 
microscópicos como parte da prática corrente nos cuidados de saúde primários (CSP). ${ }^{2}$

Deste modo, a hematúria microscópica assintomática (HMA) é encontrada de forma incidental, em cerca de 0,9\% a 18\% da população adulta. ${ }^{2}$

Na maioria das diretrizes, a HMA é definida pela presença de pelo menos três eritrócitos por campo de alta potência no estudo do sedimento urinário, devidamente colhido.

A importância deste achado é secundária ao potencial risco subjacente de patologia clinicamente significativa, incluindo malignidade (7\% a 20\% se risco elevado), ${ }^{2}$ urolitíase, infeção do trato urinário, hiperplasia benigna da próstata, patologia ginecológica e doença renal médica. ${ }^{3.5}$

É importante salientar que, embora a malignidade geniturinária tenha sido diagnosticada em aproximadamente 3\% dos doentes avaliados para HMA, o risco de detetar uma neoplasia subjacente é altamente dependente de fatores como sexo, idade, história de tabagismo e grau de hematúria. ${ }^{1}$

Enquanto a hematúria macroscópica é reconhecida como um sinal para a referenciação a consulta de urologia, a investigação recomendada da hematúria microscópica e, em particular, assintomática é inconsistente entre as diretrizes disponíveis. ${ }^{2.5}$

Como tal, há necessidade de recomendações de diretrizes atualizadas e baseadas em evidência para avaliação de hematúria que limitem os riscos e custos desnecessários associados à sobreavaliação de doentes que apresentam baixo risco de malignidade. ${ }^{1}$

Os objetivos foram rever as principais diretrizes sobre a avaliação e abordagem da HMA, criando um algoritmo de abordagem da HMA com base nas evidências mais recentes.

\section{Métodos}

Realizada uma pesquisa bibliográfica de artigos científicos, a 27 de janeiro de 2020, nas bases de dados PubMed, UpToDate e Cochrane Library, publicados nos últimos 5 anos, utilizando a combinação dos termos MeSH "Hematuria/diagnosis", em inglês e português. Incluíram-se artigos de revisão e normas de orientação clínica.

Adicionalmente, foi realizada pesquisa livre na American Urological Association (AUA) e na Associação Portuguesa de Urologia (APU).

Após realização da pesquisa bibliográfica, foram excluídos artigos duplicados e iniciada a seleção dos artigos, inicialmente por título e depois pelos resumos com base na adequação dos mesmos ao objetivo principal deste artigo. Excluídos artigos referentes à idade pediátrica.

\section{Resultados}

Foram obtidos 733 artigos da pesquisa. Após exclusão de artigos duplicados e de artigos referentes à idade pediátrica, foram sele- cionados cinco artigos de revisão, pela adequação dos mesmos ao objetivo principal.

Com base nestes, e tendo em conta a revisão sistemática mais atual (2020), que contempla as guidelines mais recentes da AUA elaborou-se um algoritmo de abordagem da HMA no adulto após estratificação do risco individual de malignidade para neoplasia urotelial (Fig. 1).

O diagnóstico de hematúria microscópica é definido pela presença de pelo menos três eritrócitos por campo de alta potência na avaliação microscópica do sedimento urinário numa amostra de urina adequadamente colhida (Recomendação Forte; Nível de Evidência: Grau C). ${ }^{1}$ De realçar que um resultado positivo em tira-teste de urina (vestígios de sangue ou superior) permanece insuficiente, dado basear-se na atividade da peroxidase, que poderá ser afetada por fatores como o uso de iodopovidona, mioglobinúria e desidratação. ${ }^{1}$ Nestes casos deve proceder-se à realização de análise sumária de urina (SU) para confirmação. (Recomendação forte; Nível de evidência: Grau C).

Perante o diagnóstico, a história clínica cuidadosa e o exame físico são recomendados por todas as diretrizes revisadas, permitindo identificar potenciais etiologias benignas que, uma vez confirmadas, podem evitar a necessidade de avaliação adicional. $^{1-3}$

$\mathrm{Na}$ anamnese devem ser identificadas potenciais causas benignas de HMA (e.g. menstruação, trauma pélvico, procedimento urológico recente, exercício físico vigoroso), considerar cuidadosamente os fatores de risco para malignidade do trato urinário (Tabela 1), e ainda outros fatores que orientem o médico para um diagnóstico específico, nomeadamente para doença renal médica e causas geniturinárias não malignas de HMA.

O médico deverá ainda avaliar a terapêutica habitual do doente, de forma a identificar fármacos potencialmente nefrotóxicos ou terapêutica antiplaquetária/anticoagulante. De referir que os médicos devem realizar a mesma avaliação em utentes com HMA que cumprem terapêutica antiplaquetária ou anticoagulante (Recomendação Forte; Nível de evidência: Grau C). ${ }^{1}$

O exame físico deve incluir a medição da pressão arterial, exame geniturinário e exame rectal no homem.

Se identificação de potenciais causas geniturinárias não malignas de HMA, a SU deverá ser repetida após resolução das mesmas (após cerca de 6 semanas). Se a HMA persistir ou a etiologia não puder ser identificada, os médicos devem realizar uma avaliação urológica baseada no risco.'

Quando a hematúria é atribuída a uma infeção do trato urinário, os médicos devem obter uma SU após o tratamento para garantir a resolução da hematúria. (Recomendação Forte; Nível de Evidência: Grau C). ${ }^{1}$

Laboratorialmente, é recomendada, além da SU, a determinação da função renal (creatinina, ureia, taxa de filtração glomerular estimada e proteinúria) na exclusão de patologia nefro- 
Hematúria Microscópica Assintomática: Qual a Melhor Forma de Abordagem?

\section{HEMATÚRIA MICROSCÓPICA ASSINTOMATICA (HMA)}

\section{( $\geq 3$ eritrocitos/campo na SU)}

\section{HISTÓRIA CLÍNICA}

- Fatores de risco para malignidade trato urinário

- Potenciais etiologias (ex, infeção, menstruação, trauma pélvico, exercicio fisico vigoroso ou procedimento urológico recente)

Terapêutica habitual (nefrotoxicidade, anticoagulantes, antiplaquetários)

EXAME Físıco (TA, exame geniturinário, exame rectal no homem)

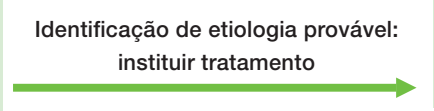

su

(após 6 semanas)

Consulta de Nefrologia
se proteinuria de novo,
eritrocitos dismórficos, cilindros
celulares ou insuficiência renal

\section{Controlo Analítico |TFG estimada}

Função renal (creatinina, ureia, proteinuria)

celulares ou insuficiência renal

\section{ESTRATIFICAÇÃO DO RISCO}

Repetição SU

Positiva

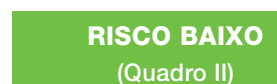

(Quadro II)

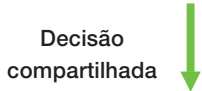

Repete SU em 6 meses

OU

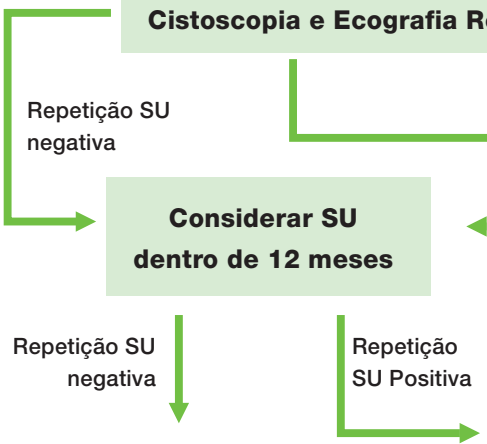

Suspende follow-up



RISCO INTERMÉDIO

(Quadro II)

Cistoscopia e

Ecografia Renal
RISCO ALTO

(Quadro II)
Repetiçăo SU Positiva

Repetição SU negativa



istoscopia e

Uro-TC

Tratamento conforme indicado

Se diagnostico urológico não maligno, repetir $\mathrm{AU}$ após tratamento

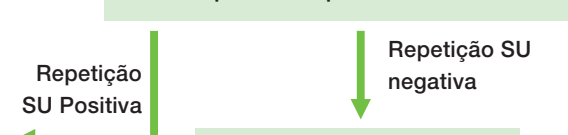

Suspende follow-up vs observação

Considerar a imagem transversal com urografia ou pielogramas retrógrados, se não realizados anteriormente

\section{Reavaliação}

Se aparecimento hematuria macroscópica, aumentar o grau de HMA ou sintomas urológicos de novo

Figura 1 - Proposta de algoritmo de abordagem da HMA no adulto

SU - análise sumária de urina; TA - tensão arterial; TFG - Taxa de filtração glomerular; Uro-TC - urografia por tomografia computorizada 


\section{FATORES DE RISCO}

- Sexo masculino

- Idade (> 50 anos)

- Fumador atual ou ex-fumador

- Exposição ocupacional ou outra a químicos/corantes (hidrocarbonetos aromáticos policíclicos ou aminas aromáticas +

- Abuso de analgésicos

- Grau de hematúria microscópica

- HMA persistente

- História de:

- Hematúria macroscópica

- Doença ou alteração urológica

- Sintomas de irritação miccional (urgência e frequência)

- Radiação pélvica

- Infeção crónica do trato urinário

- Exposição a agentes carcinogénicos conhecidos ou quimioterapia tal como agentes alquilantes (particularmente a ciclofosfamida)

- Presença de corpo estranho cronicamente

- História familiar de neoplasia urotelial ou síndrome de Lynch.

\section{Tabela 1. Fatores de risco comuns para neoplasia do trato urinário ${ }^{1,2}$}

* Limpa-chaminés, enfermeiros, empregado de mesa, trabalhadores no alumínio, navios ou óleo/petróleo

+ Tabaco, corantes, trabalhadores do couro ou de impressões, cabeleireiros

lógica. ${ }^{3}$ Utentes com proteinúria de novo, eritrócitos dismórficos, cilindros celulares ou insuficiência renal devem ser referenciados a consulta de nefrologia, contudo isso não exclui a necessidade de avaliação urológica baseada no risco para identificar patologia urológica coexistente.

Após esta a avaliação inicial, o médico deve categorizar o doente que apresente HMA em baixo, intermédio ou alto risco para patologia maligna (Tabela 2). (Recomendação Forte; Nível de Evidência: Grau C).

Se baixo risco, os médicos devem envolver os doentes na tomada de decisão compartilhada para decidir entre repetir a SU em seis meses ou prosseguir com a cistoscopia e ultrassonografia renal. (Recomendação Moderada; Nível de Evidência: Grau C). A probabilidade de diagnosticar patologia maligna é baixa, portanto devem ser discutidos e ponderados benefícios e potenciais malefícios na investigação com cistoscopia e ecografia, incluindo a deteção de falsos positivos. Doentes que inicialmente optaram por repetição de SU em 6 meses, e voltem a apresentar HMA devem ser reclassificados como de risco intermédio ou alto, pelo que deverão realizar cistoscopia e imagem do trato urinário superior de acordo com as recomendações para essas categorias de risco. (Recomendação Forte; Nível de Evidência: Grau C). ${ }^{1}$

Se doente com HMA categorizado como de risco intermédio para malignidade, é aconselhável a realização de cistoscopia e ultrassonografia renal. (Recomendação Forte; Nível de Evidência: Grau C), realçando que a cistoscopia apresenta uma sensibilidade de $98 \%$ na identificação de neoplasia vesical, contrariando a baixa sensibilidade da imagem ecográfica para tal. Em relação à escolha da imagem do trato urinário superior, a ecografia renal tem sensibilidade e especificidade adequadas para tumores corticais renais em comparação com urografia por tomografia computorizada (Uro-TC), com menor custo e risco.

Doentes com HMA categorizados como de alto risco para malignidade, deverão realizar cistoscopia e Uro-TC. (Recomendação Forte; Nível de Evidência: Grau C).

Doentes com HMA e história familiar de carcinoma de células renais ou síndromes genéticas de tumores renais, deverão realizar exames de imagem do trato urinário superior, independentemente da categoria de risco. ${ }^{1}$

Quando a investigação da HMA se revela negativa, ponderar SU dentro de 12 meses (Nível de evidência: Grau C). Se esta negativa deverá ser suspensa avaliação adicional para HMA (Nível de evidência: Grau C), contudo é importante salientar que as mudanças no estado clínico do doente, particularmente o desenvolvimento de hematúria macroscópica, devem levar à revisão clínica.

\section{Discussão}

As diretrizes atuais variam em relação a detalhes importantes como a definição de HMA e o método ideal para avaliação radiológica (Tabela 3), refletindo a ausência de evidências robustas sobre o assunto. ${ }^{1-3}$

Contudo, podemos verificar que existe consenso pela maioria das diretrizes que consideram inadequado o diagnóstico de HMA com base exclusivamente no teste urinário com fita-reagente, 


\begin{tabular}{|c|c|c|}
\hline $\begin{array}{c}\text { Risco Baixo }<1 \% \\
\text { (o doente cumpre todos os critérios) }\end{array}$ & $\begin{array}{l}\text { Risco Intermédio - 1\% - 2\% } \\
\text { (o doente cumpre um destes critérios) }\end{array}$ & $\begin{array}{c}\text { Risco Alto }>10 \% \\
\text { (o doente cumpre um destes critérios) }\end{array}$ \\
\hline $\begin{array}{l}\text { - Mulher }<50 \text { anos; Homem }<40 \text { anos } \\
\text { - Não-fumador ou fumador }<10 \text { UMA } \\
\text { - 3-10 Erit/campo numa amostra única de urina II } \\
\text { - Ausência fatores de risco para neoplasia urotelial }\end{array}$ & $\begin{array}{l}\text { - Mulher 50-59 anos; Homem 40-59 anos } \\
\text { - Fumador 10-30 UMA } \\
\text { - 11-25 Erit/campo numa amostra única de urina II } \\
\text { - Doente de baixo risco sem avaliação prévia com } \\
\text { 3-10 Erit/campo na repetição de urina II } \\
\text { - Fatores de risco adicionais para neoplasia urotelial }\end{array}$ & $\begin{array}{l}\text { - Mulher ou Homem > } 60 \text { anos } \\
\text { - Fumador r>30 UMA } \\
\text { - }>25 \text { Erit/campo numa amostra única de } \\
\text { Urina II } \\
\text { - História de hematúria macroscópica }\end{array}$ \\
\hline
\end{tabular}

Tabela 2. Sistema de estratificação do risco para neoplasia urotelial na presença de HMA

(Adaptado de Barocas DA, et al. Microhematuria: AUA/SUFU Guideline. J Urol. 2020;204:778-86. ${ }^{1}$ )

Erit - eritrócitos; UMA - unidades maço ano

dada a existência de uma percentagem importante de falsospositivos. Deste modo, as diferentes organizações recomendam a avaliação do sedimento urinário para diagnóstico de hematúria microscópica. Em contrapartida, não são unânimes quanto à necessidade de apenas uma amostra de urina para estabelecimento do diagnóstico. A revisão da literatura da diretriz da AUA de 2012 e dados mais recentes apoiam a definição de hematúria microscópica na avaliação microscópica de uma única amostra de urina, enfatizando a importância de recomendar laboratórios que relatam o grau de hematúria quantitativamente (eritrócitos/ /campo de maior ampliação).

Após estabelecimento do diagnóstico e avaliação cuidadosa do doente, é fundamental a exclusão de etiologias potencialmente benignas de HMA antes de prosseguir com a investigação segundo o algoritmo.

$\mathrm{Na}$ avaliação da HMA, podemos verificar que as diretrizes são consensuais relativamente ao papel da cistoscopia. ${ }^{2}$ Deste modo, e seguindo o protocolo de abordagem, quando se opta pela realização de cistoscopia o doente deverá ser referenciado a consulta hospitalar de urologia para a sua realização, dado este não ser um exame da prática corrente dos CSP.

Relativamente à avaliação radiológica, há vários anos que tem vindo a ser discutida pelas diferentes entidades a importância da estratificação do risco para doença neoplásica do trato urinário na escolha do método de imagem mais apropriado. Algumas diretrizes defendem que esta escolha deve ser determinada pelos recursos disponíveis, vontade do doente e situação clínica. Contudo, uma avaliação mais limitada poderá ser suficiente em doentes de baixo risco, evitando riscos desnecessários para o utente. ${ }^{1}$ É com base neste princípio que foi elaborado este protocolo de abordagem, permitindo a orientação pelo médico de família de uma parte das situações.

Importa ainda reforçar que na suspeita de etiologia nefrológica o doente deverá ser orientado a consulta da especialidade respetiva, no entanto tal não exclui a necessidade de avaliação urológica baseada no risco.

Apesar do nível baixo de evidência das diferentes diretrizes, a HMA representa uma valiosa oportunidade de para futuras

\begin{tabular}{|c|c|c|c|c|c|c|c|}
\hline \multirow[t]{2}{*}{ Recomendação } & \multirow[t]{2}{*}{ Ano } & \multirow[t]{2}{*}{ Referência } & \multicolumn{3}{|c|}{ Definição de HMA } & \multicolumn{2}{|c|}{ Componentes da avaliação } \\
\hline & & & $\begin{array}{l}\text { Tira-teste } \\
\text { Urinária }\end{array}$ & $\begin{array}{l}\text { Resultado de } \\
\text { sedimento urinário } \\
\text { Eritrócitos/campo } \\
\text { grande ampliação }\end{array}$ & $\begin{array}{l}\text { Amostras de } \\
\text { urina com } \\
\text { resultado } \\
\text { positivo }\end{array}$ & Cistoscopia & $\begin{array}{c}\text { Método de imagem } \\
\text { preferido }\end{array}$ \\
\hline $\begin{array}{l}\text { American Urological Association } \\
\text { guideline }\end{array}$ & 2020 & 1 & Inadequado & $\geq 3$ & 1 & Todos os doentes & $\begin{array}{l}\text { Segundo categoria } \\
\text { de risco }\end{array}$ \\
\hline $\begin{array}{l}\text { Associação Portuguesa } \\
\text { de Urologia }\end{array}$ & & 7 & Inadequado & $\geq 3$ & $2 / 3$ & $\begin{array}{l}\text { Consoante a citologia/ } \\
\text { risco malignidade }\end{array}$ & $\begin{array}{c}\text { Ecografia renal } \\
\text { (ponderar Uro-TC) }\end{array}$ \\
\hline $\begin{array}{l}\text { Canadian Urological Association } \\
\text { guideline }\end{array}$ & 2008 & 2 & Inadequado & $\geq 2$ & 2 & Todos os doentes & Ecografia renal \\
\hline $\begin{array}{l}\text { British Association of Urological } \\
\text { Surgeons guideline }\end{array}$ & 2008 & 2 & $\geq 1$ heme & Não requerida & $2 / 3$ & Não específica & Não específica \\
\hline Dutch Guideline on Hematuria & 2010 & 2 & Inadequado & $\geq 3$ & $2 / 3$ & Todos os doentes & Ecografia renal \\
\hline
\end{tabular}

Tabela 3. Recomendações organizacionais para avaliação da HMA 
investigações, de forma a melhorar significativamente a abordagem do doente com HMA. ${ }^{1-3,5}$

Essas áreas incluem o uso de novos instrumentos automatizados para SU, validação de grupos de risco, utilidade de biomarcadores urinários e cistoscopia aprimorada para HMA, aperfeiçoamento de técnicas de imagem para reduzir a exposição à radiação e investigação adicional da história natural de doentes com HMA após avaliação negativa. ${ }^{1}$

\section{Conclusão}

Devido à combinação de uma prevalência relativamente alta de HMA na população adulta com uma baixa prevalência de doença clinicamente significativa, este artigo visa apoiar a avaliação e abordagem da HMA no adulto, fornecendo uma estratégia baseada no risco individual de cada doente fundamentada na evidência mais atual.

Os médicos responsáveis pela abordagem da HMA devem ter em consideração as variações nos recursos disponíveis e as tolerâncias, necessidades e preferências dos doentes, devendo ser discutida a dicotomia risco-benefício da abordagem proposta previamente à decisão clínica.

\section{Responsabilidades Éticas}

Conflitos de Interesse: Os autores declaram não possuir conflitos de interesse.

Suporte Financeiro: O presente trabalho não foi suportado por nenhum subsidio o bolsa ou bolsa.

Proveniência e Revisão por Pares: Não comissionado; revisão externa por pares.

\section{Ethical Disclosures}

Conflicts of Interest: The authors have no conflicts of interest to declare.

Financial Support: This work has not received any contribution grant or scholarship.
Provenance and Peer Review: Not commissioned; externally peer reviewed.

\section{*Autor Correspondente/Corresponding Author:}

Nome: Diana Cláudia Duarte da Rocha

Endereço eletrónico: dianarocha91@gmail.com

Endereço Postal: Avenida da Câmara, 1, Moure, 4730 - 300 VILA

VERDE, Braga, Portugal

Recebido/Received: 2020-11-12

Aceite/Accepted: 2021-09-10

Publicado /Published: 2022-01-25

(C) Author(s) (or their employer(s)) and Acta Urológica Portuguesa 2021. Re-use permitted under CC BY-NC. No commercial re-use. (C) Autor (es) (ou seu (s) empregador (es)) e Acta Urológica Portuguesa 2021. Reutilização permitida de acordo com CC BY-NC. Nenhuma reutilização comercial.

\section{Referências}

1. Barocas DA, Boorjian SA, Alvarez RD, Downs TM, Gross CP, Hamilton BD, Kobashi KC, Lipman RR, Lotan Y, Ng CK, Nielsen ME, Peterson AC, Raman JD, Smith-Bindman R, Souter LH. Microhematuria: AUA/SUFU Guideline. J Urol. 2020;204:778-86. doi: 10.1097/JU.0000000000001297.

2. Linder BJ, Bass EJ, Mostafid H, Boorjian SA. Guideline of guidelines: asymptomatic microscopic haematuria. BJU Int. 2018;121:176-83. doi: 10.1111/bju.14016.

3. Ziemba J, Guzzo, TJ, Ramchandani P. Evaluation of the patient with asymptomatic microscopic hematuria. Acad Radiol. 2015; 22:10347. doi: 10.1016/j.acra.2015.02.003.

4. Niemi MA, Cohen RA. Evaluation of microscopic hematuria: a critical review and proposed algorithm. Adv Chronic Kidney Dis. 2015; 22:289-96. doi: 10.1053/j.ackd.2015.04.006.

5. Reis F, Silva J, Silva C. Algoritmos de decisão em Urologia: Avaliação de hematúria não traumática assintomática no adulto. Acta Urol Port. 2006. 23: Separata. [consultado Maio 2020] Disponível em https:// apurologia.pt/wp-content/uploads/2019/04/hematuria-1.pdf 